\title{
Clinical Predictors of In-Hospital Outcomes in COVID-19 Patients: A Retrospective Cohort Study
}

\author{
Maiara A Floriani, MSc ${ }^{1 *}$ (D) Marina Bessel, ScD², Isabelle W Zorzo, BSHCA ${ }^{1}$, Andressa B Glaeser,

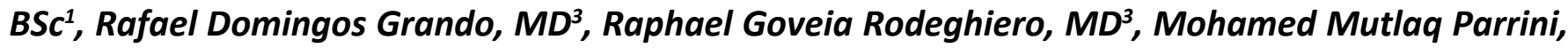 \\ BEcon, MPhil ${ }^{4}$, Gisele Nader Bastos, MD, ScD ${ }^{5}$ and Luiz Antonio Nasi, MD, ScD 6
}

${ }^{1}$ Value Management Office, Hospital Moinhos de Vento, Porto Alegre, Rio Grande do Sul, Brazil

${ }^{2}$ PROADI-SUS Project Office, Hospital Moinhos de Vento, Porto Alegre, Rio Grande do Sul, Brazil

${ }^{3}$ Department of Radiology, Hospital Moinhos de Vento, Porto Alegre, Rio Grande do Sul, Brazil

${ }^{4}$ Cheif Executive Officer, Hospital Moinhos de Vento, Porto Alegre, Rio Grande do Sul, Brazil

${ }^{5}$ Medical Manager, Hospital Moinhos de Vento, Porto Alegre, Rio Grande do Sul, Brazil

${ }^{6}$ Cheif Medical Officer, Hospital Moinhos de Vento, Porto Alegre, Rio Grande do Sul, Brazil

*Corresponding author: Maiara Anschau Floriani, MsC, Head of Value Management Office, Hospital Moinhos de Vento, Moinhos Research Institute, 910 Ramiro Barcelos Street, $5^{\text {th }}$ Floor, Porto Alegre-RS-90560-030, Brazil, Tel: +55-51-33143207

\begin{abstract}
Background: We do not have detailed evidence on conditions or treatments that act as predictors of hospital outcomes.

Objective: To identify the clinical predictors of hospital outcomes in COVID-19 patients and to evaluate the effect of therapeutic's interventions on length of stay, ICU admission, need for mechanical ventilation (MV) and mortality.

Materials and methods: The primary outcome was to assess whether any treatment alone or in combination with standard care, was able to reduce ICU admission, need for MV or mortality. The secondary end points were 1) To know if any single or combined treatment was able to reduce the length of stay in the hospital, MV and ICU, 2) Whether some form of oxygen support prevented MV and 3) Which clinical predictors are relevant for worse outcome.

Results:AZM-Corticosteroidand therapeuticanticoagulation when indicated reduced the ICU stay in 1.5 day and MV in 4 days, but when the same combination included HCQ this time increased. For the AZM-Corticosteroid and therapeutic anticoagulation combination the mean ICU length stay was 15.9 days, however when $\mathrm{HCQ}$ was present, again the mean ICU time was 40.3 days. The clinical predictors at admission for death were: Age > 65 years, presence of up one comorbidity, pulmonary involvement more than $50 \%$,
\end{abstract}

saturation < 93\%, lymphocytes < $900 \mathrm{~mm}^{3}$, D-dimers > $1.250 \mathrm{ng} / \mathrm{mL}$ and C-reactive protein (CRP) $>8.0 \mathrm{mg} / \mathrm{dL}$.

Conclusion: AZM-Corticosteroids and therapeutic anticoagulation, when indicated, represented a favorable combination for inpatients with COVID-19.Some clinical predictors at admission may help to estimate a higher risk of poor evolution.

\section{Keywords}

Coronavirus infections, Therapeutics, Cohort studies, Risk factors, Hydroxychloroquine, Azithromycin

\section{Introduction}

Doctors and hospitals have learned a lot about how best to treat people infected with the novel coronavirus disease 2019 (COVID-19) in the last months since the pandemic began. A significant percentage of patients develop health conditions that require hospital care [1]. Some conditions prior to hospitalization have been presented with an important role in the hospitalization flow. However, we still do not have detailed evidence on conditions or treatments that act as clinical predictors of hospital outcomes in COVID-19 patients.

Citation: Floriani MA, Bessel M, Zorzo IW, Glaeser AB, Grando RD, et al. (2021) Clinical Predictors of In-Hospital Outcomes in COVID-19 Patients: A Retrospective Cohort Study. Int Arch Intern Med 5:035. doi.org/10.23937/2643-4466/1710035

Accepted: December 29, 2021: Published: December 31, 2021

Copyright: (c) 2021 Floriani MA, et al. This is an open-access article distributed under the terms of the Creative Commons Attribution License, which permits unrestricted use, distribution, and reproduction in any medium, provided the original author and source are credited. 
The practice has varied widely across the world and several therapeutic interventions have been proposed and methodological studies have been published, although far from overwhelming evidence they closely follow and analyze updates on this outbreak [2]. Therapeutic strategies using hydroxychloroquine (HCQ), antibiotics, corticosteroid, anticoagulants and others, in combination or not, were introduced to the clinical practice. However, there is not consensus about the best pharmacological combination in the usual care in the COVID-19 treatment.

Our aim was to identify the main clinical predictors for a worse prognosis during COVID-19 hospitalization and to evaluate retrospectively the medical practice in the real world of our hospital, considering the main therapeutics' interventions on length of hospital stay, need for admission to the intensive care unit (ICU) or mechanical ventilation (MV) and mortality during the COVID-19 outbreak in our center.

\section{Methods}

\section{Study design and participants}

The study was approved by the institutional ethics committee. This was a single-center, retrospective, observational cohort study between March 15, 2020 and July 31, 2020 at Hospital Moinhos de Vento, a teaching private hospital, reference for the treatment of patients with COVID-19 in the South of Brazil [3]. Patients admitted (aged $\geq 18$ years) with RT-PCR positive for COVID-19 obtained from nasal and pharyngeal swabs were consecutively recruited.

Patients were assessed retrospectively for specific outcomes after receiving a set of therapeutic interventions. The interventions in different combinations, besides the usual care were: $\mathrm{HCQ}$, azithromycin (AZM), corticosteroids (dexamethasone, hydrocortisone or methylprednisolone), tocilizumab, oseltamivir, convalescent plasma, therapeutic anticoagulation and different ways of improving oxygen without positive pressure (nasal catheter and Hudson mask) and with positive pressure (high flow nasal cannula (HFNC) Bi-level Airway Positive Pressure (BiPAP) and MV). Therapeutic anticoagulation was considered with intention-to-treat for patients with Deep Venous Thrombosis (DVT), Pulmonary Embolism (PE) or serum D-dimers $>2,000 \mathrm{ng} /$ $\mathrm{mL}$. Obese people (body mass index $\geq 30$ ), lymphopenia (lymphocytes $<900 \mathrm{~mm}^{3}$ ), D-dimers $>1,250 \mathrm{ng} / \mathrm{mL}$ and C-reactive protein (CRP) $>8.0 \mathrm{mg} / \mathrm{dL}$ were considered abnormal values. The usual dose of corticosteroids used was $\leq 0.5-1 \mathrm{mg} / \mathrm{kg}$ per day of methylprednisolone or equivalent. The pulmonary impairment was assessed on a visual scale by two independent chest radiologists trained to interpreted COVID-19 patients.

The primary endpoint was building up a COVID-19 predictor model based on individual-level data to estimate ICU admission, need for MV and hospital mortality. Additionally, three secondary endpoints were established: 1) The impact of these interventions on the length of stay in hospital, ICU and MV; 2) The likelihood of the different ways of noninvasive oxygen administration to prevent $\mathrm{MV}$ and 3) Which clinical predictors are relevant to poor outcome (mortality).

\section{Statistical analysis}

Descriptive analysis was used to characterize the study population. Categorical variables weresummarized using absolute frequencies and percentages, while continuous variables were analyzed using means and standard deviation (SD) or median and interquartile range (IQR). Different combinations of medications were administered and the one used in multivariable models was the use of AZM and corticosteroids with or without the use of therapeutic anticoagulation. Interaction term with HCQ was used to evaluate the behavior of the combined therapy of medications in the presence or absence of the use of $\mathrm{HCQ}$.

Logistic regression analysis was conducted to examine factors associated with the outcomes, adjusting for all predictors presented in the model. Linear regression was applied to continuous outcomes. The model results are presented in odds ratio (OR) or beta $(\beta)$. For the multivariable analyses, a theoretical framework was structured according to literature. Statistical analysis was performed using SAS software (Statistical Analysis System, SAS Institute Inc., Cary, N.C.), version 9.4, and statistical significance was defined as $p$-value $<0.05$.

\section{Results}

Between March 15 and July 31, 2020, 459 patients who had been admitted to Hospital Moinhos de Vento were identified and considered to meet the criteria.

\section{Clinical characteristics}

The median age was 60.0 years (interquartile range [IQR], 45.0 to 72.0 years) being $262(57.1 \%)$ males. The main clinical characteristics of patients are presented in Table 1 and Table 2. The median onset of symptoms before admission was 7.0 days (IQR, 4.0 to 9.5 days); 136 (29.6\%) patients needed admission to the ICU and $97(21.1 \%)$ required MV. The mean length of stay in hospital, MV and ICU was 13.9 (SD \pm 16.1 ), 20.1 (SD \pm $15.6)$ and $21.2(S D \pm 18.4)$ days, respectively.

\section{Therapeutic's interventions}

Regarding pharmacologic therapies, HCQ-AZM was administered to 105 (23.2\%) patients; HCQcorticosteroid to 49 (10.7\%) and AZM-corticosteroid to 212 (46.8\%). Therapeutic anticoagulation was administered to $138(31.8 \%)$ patients, being 36 (7.9\%) combined with $\mathrm{HCQ}$ and 78 (17.2\%) with AZM-Corticosteroid. The combination of HCQ-AZMCorticosteroid and therapeutic anticoagulation occurred in 21 patients $(4.6 \%)$. 
Table 1: Clinical information at admission.

\begin{tabular}{|c|c|c|c|}
\hline$n=459$ & n (\%) or Median [IQR] & & n (\%) or Median [IQR] \\
\hline \multicolumn{2}{|l|}{ Demographics } & \multicolumn{2}{|l|}{ Compromise Lung (Chest CT) } \\
\hline Male, age $(y)$ & $\begin{array}{l}262(57.0) ; 60.0 \\
{[45.0-72.0]}\end{array}$ & $<50 \%$ & $351(83.7)$ \\
\hline Obesity BMI $\geq 30$ & $148(33.1)$ & $\geq 50 \%$ & $68(16.2)$ \\
\hline \multicolumn{2}{|l|}{ Comorbidity } & \multicolumn{2}{|l|}{ Laboratory } \\
\hline Hypertension & $214(46.8)$ & Hemoglobin (12.0-15.5 g/dL) & $13.7[12.5-14.8]$ \\
\hline $\begin{array}{l}\text { Pulmonary disease (emphysema, } \\
\text { COPD, asthma) }\end{array}$ & $101(22.1)$ & Leukocytes (> 5.000\%/mm³) & $286(62.7)$ \\
\hline Diabetes & $99(21.6)$ & Lymphocytes (900-2.900 mm³) & $1.030[755-1.370]$ \\
\hline Chronic kidney disease & $43(9.4)$ & Platelets $\left(150.000-450.000 \mathrm{~mm}^{3}\right)$ & $\begin{array}{l}177.000 \\
{[140.000-225.000]}\end{array}$ \\
\hline Cancer (last 5 y) & $43(9.4)$ & Creatinine $(0.60-1.10 \mathrm{mg} / \mathrm{dL})$ & $0.95[0.81-1.16]$ \\
\hline \multicolumn{2}{|l|}{ Self-Reported Symptoms } & C-reactive protein (> $8.0 \mathrm{mg} / \mathrm{dL}$ ) & $141(33.6)$ \\
\hline Fever & $284(63.5)$ & D-dimers (> 1.250 ng/mL FEU) & $71(17.5)$ \\
\hline Cough & $271(60.6)$ & Lactate dehydrogenase (240-480) & 554 [443-680] \\
\hline Dyspnea & $173(38.7)$ & Total bilirubin $(0.0-0.30 \mathrm{mg} / \mathrm{dL})$ & $0.38[0.27-0.49]$ \\
\hline Headache & $130(29.0)$ & Troponin (under 14 ng/L) & $7.0[5.0-13.0]$ \\
\hline Muscle pain (myalgia) & $117(26.1)$ & BNP (up to $100 \mathrm{pg} / \mathrm{mL}$ ) & $33.0[13.0-192.0]$ \\
\hline Sore throat & $90(20.1)$ & IL-6 (range $1.5-7.0 \mathrm{pg} / \mathrm{mL}$ ) & $37.0[9.4-61.0]$ \\
\hline Fatigue & $84(18.7)$ & & \\
\hline Coryza & $75(16.7)$ & & \\
\hline Diarrhea & $54(12.0)$ & & \\
\hline Nausea and Vomiting & $53(11.8)$ & & \\
\hline Presence of up to 3 symptoms & $251(56.1)$ & & \\
\hline
\end{tabular}

Y: Year; BMI: Body mass index; COPD: Chronic obstructive pulmonary disease; CT: Computed tomography; BNP: Brain natriuretic peptide

Table 2: Clinical information at hospitalization.

n (\%) or median [IQR]

Onset of symptoms and hospital admission 7.0 [4.0-9.5] (days)

\section{Oxygen supplementation}

Nasal catheter (NC)

Hudson mask

Noninvasive ventilation (NIV)

High flow nasal cannula (HFNC)

Mechanical ventilation (MV)

Oxygen therapy combination

$\mathrm{NC} \rightarrow$ Hudson mask

$79(17.7)$

$\mathrm{NC} \rightarrow$ Hudsonmask $\rightarrow$ NIV

$48(10.8)$

$\mathrm{NC} \rightarrow$ Hudsonmask $\rightarrow$ NIV $\rightarrow$ HFNC

$26(5.8)$

$23(5.1)$

MV

Intensive care unit (ICU)

ICU length of stay (days)

With MV

Without MV

\begin{tabular}{|l|}
\hline $247(55.5)$ \\
\hline $86(19.3)$ \\
$76(17.0)$ \\
$62(13.9)$ \\
$97(21.1)$ \\
\hline $79(17.7)$ \\
\hline $48(10.8)$ \\
\hline $26(5.8)$ \\
\hline $23(5.1)$ \\
\hline $136(29.6)$ \\
\hline $15.1[7.1-29.5]$ \\
\hline $25.2[11.1-37.0]$ \\
\hline $7.2[5.3-10.5]$ \\
\hline
\end{tabular}

\section{Pharmacological treatments}

Antibiotic (except azithromycin) 359 (79.7)

\begin{tabular}{l|l} 
Azithromycin & $340(75.0)$
\end{tabular}

Corticosteroid 283 (61.9)

\begin{tabular}{l|l} 
Anticoagulant $^{*}$ & $138(31.8)$
\end{tabular}

\begin{tabular}{l|l} 
Antiviral (oseltamivir) & $130(29.6)$
\end{tabular}

Hydroxychloroquine $\quad 115(25.1)$

Convalescent plasma $\quad 76$ (16.5)

Vasopressor $\quad 69(15.7)$

Anti-IL6 drugs (tocilizumab) 12 (2.6)

Azithromycin + Corticosteroid $116(25.6)$

Azithromycin + Corticosteroid + 78 (17.2)

Anticoagulant

\section{Outcomes}

Hospital discharge $398(86.7)$

Death $40(8.7)$

Censored $^{* *} \quad 21(4.5)$

ICU: Intensive care unit; MV: Mechanical ventilation; "Anticoagulation used with treatment intention; "*Censored means patients without outcome results 
Predictor to outcomes

Odds Ratio

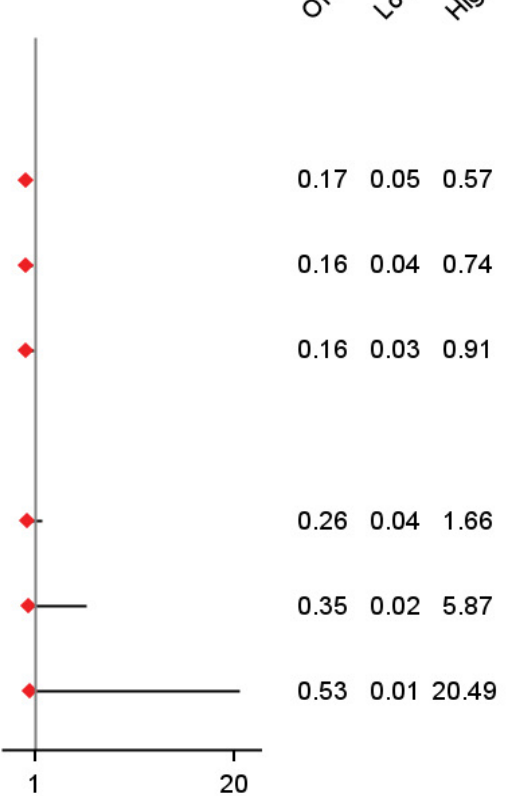

Odds Ratio and Cl95'

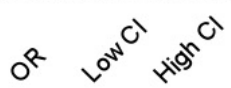

Azithromycin+Corticosteroid without HCQ

ICU admission

Need for mechanical ventilation

Mortality

Azithromycin+Corticosteroid with HCQ

ICU admission

Need for mechanical ventilation

Mortality

20

Figure 1: Risk predictors to primary endpoint-ICU admission, need for MV and hospital mortality.

For patients treated with AZM-Corticosteroid the risk for ICU admission 0.17 (OR; $95 \% \mathrm{Cl} 0.05$ to 0.57 ), for MV 0.16 (OR; 95\% Cl 0.04 to 0.74 ) and mortality was 0.16 (OR; $95 \% \mathrm{Cl} 0.03$ to 0.91 ). For those patients treated with HCQ-AZM-Corticosteroid, the association represented loss of benefit (Figure 1). Figure 1 Outcomes risk according to treatment combined.

Tocilizumab (2.6\%), convalescent plasma (16.5\%) and oseltamivir (29.6\%) were administered as adjunct therapy. Among patients admitted to the ICU, 57.6\% (68) received vasopressor and supplemental oxygen without positive pressure was used in $56.9 \%$ (254). Positive non-invasive ventilation including HFNC and BiPAP were used in $23.9 \%$ (107) while MV in 21.1\% (97). HFNC oxygen therapy was able to prevent the patient's progression to MV in $31.8 \%$ of cases.

\section{Main outcomes and endpoints}

Overall, $86.7 \%$ (398) of the patients were discharged alive and $4.5 \%$ (21) were still hospitalized by dataset freeze date. Of patients admitted to the ICU, 25.7\% (35) died and when $\mathrm{MV}$ was required, the mortality increased to $34.0 \%$ (33). Among dead patients, most had median age of 83.3 years (IQR, 75.5 to 89.5 years) with mean length of stay in hospital of 25.3 (SD \pm 22.5 ) days, in ICU 22.8 (SD \pm 18.7 ) and in MV 21.2 (SD \pm 17.3). The main clinical predictors related to increased mortality, with $>70 \%$ risk, were: Age $>65$ years, presence of up one comorbidity, pulmonary involvement $>50 \%$, saturation $<93 \%$, lymphocyte $<900 \mathrm{~mm}^{3}$, D-dimers $>1,250 \mathrm{ng} / \mathrm{mL}$ and $\mathrm{CRP}>8.0 \mathrm{mg} / \mathrm{dL}$ at admission, Oxygen requirement through BiPAP or HFNC, and ICU admission and MV required during hospitalization were also associated with a higher risk of death (Figure 2).
Considering the secondary endpoints, AZMCorticosteroid decreased the mean length of hospital stay in 1.5 day ( $\beta-1.595 \% \mathrm{Cl}-4.9$ to 1.9 ), while $\mathrm{HCQ}$ alone increased in 6.9 days $(\beta 6.995 \% \mathrm{Cl} 3.6$ to 10.3$)$. AZM-Corticosteroid and therapeutic anticoagulation combination reduced the ICU length stay in 1.5 day ( $\beta$ $-1.595 \% \mathrm{Cl}-9.7$ to 6.7 ) and $\mathrm{MV}$ in 4 days ( $\beta-4.095 \%$ $\mathrm{Cl}-13.4$ to 5.3 ), however, this effect was not observed when HCQ was associated (Figure 3). For the AZMCorticosteroid and therapeutic anticoagulation the mean length of ICU stay was 15.9 days, but when HCQ was included, again this time was increased to 40.3 days (Figure 3). There was a trend to more time in MV in obese patients. All models were adjusting for sex, age, obesity, pulmonary involvement, D-dimers, CRP, oxygen support without positive pressure (nasal catheter and Hudson mask) and length of hospital stay. Regarding HCQ have been used or not, the comparability of the groups was verified in relation to the clinical predictors for mortality, where there was no difference was observed among those who received the drug or not between those treated or not with HCQ. Figure 3 Risk factors associated with length of stay.

\section{Discussion}

Our study sought to identify the clinical predictors for COVID-19 that resulted in risk for worst outcomes and the effect of a set of therapeutic interventions on length of stay, ICU admission, need for MV and mortality. The study was conducted based in a real world of care at a Brazilian private hospital. Our major findings suggest: 1) There was a benefit with the combination of AZMCorticosteroid to reduce the risk of ICU admission, need for MV and mortality; 2) The combination of 


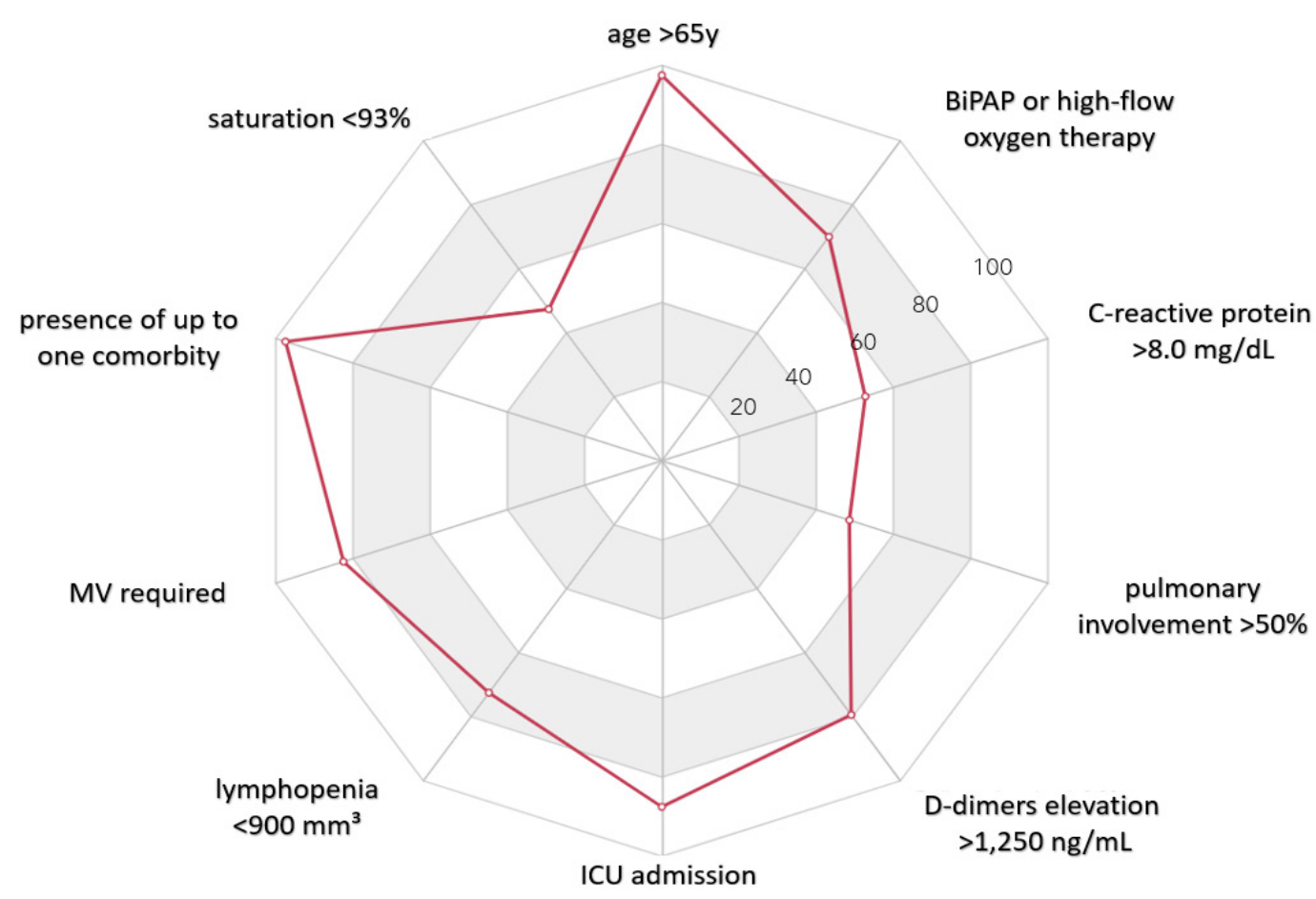

Figure 2: Clinical predictors in mortality.

Predictor to length of stay

Male sex

More than 65 years

obesity

At least one comorbidity

Chest Computed Tomography $>50 \%$

C-reactive protein $>8$

D-Dimers > 1250

Nasal oxygen catheter

Hudson mask

Azithromycin+Corticosteroid

Hydroxych loroquine

Azithromycin+Corticosteroid ${ }^{\star}$ Hydroxych loroquine

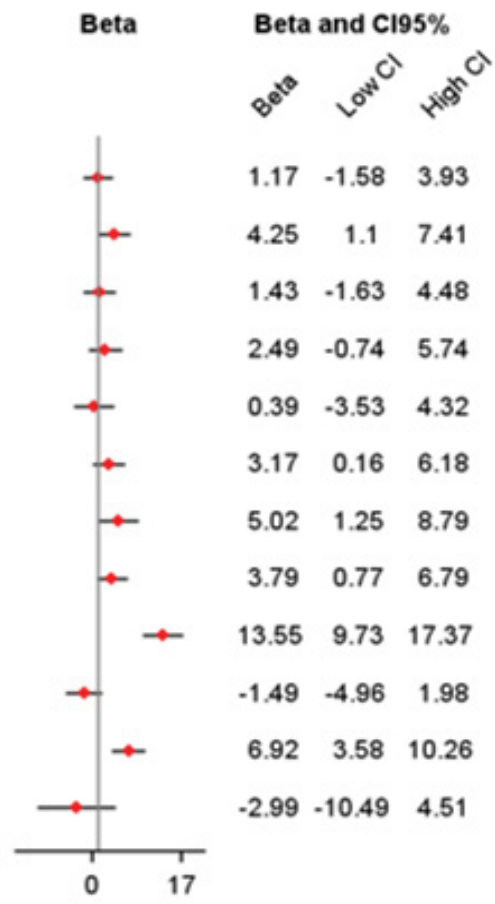


Predictor to length of stay in ICU

Male sex

More than 65 years

obesity

At least one comorbidity

chest computed Tomography $>50 \%$

C-reactive protein $>8$

D-Dimers > 1250

Nasal oxygen catheter

Hudson mask

Azithromycin+Corticosteroid+Anticoagulant Hydroxych loroquine

Medication interaction
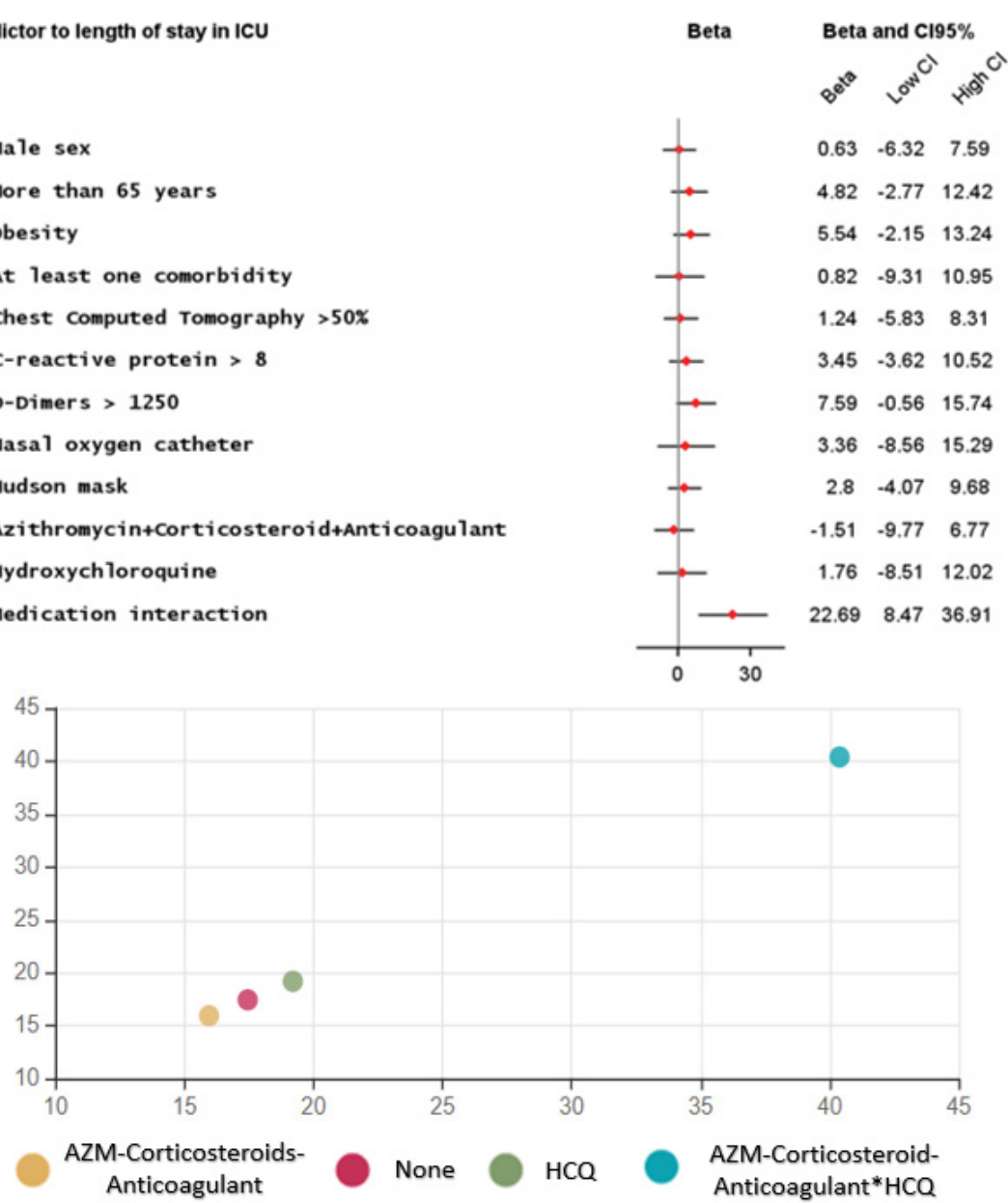

At least one comorbidity

Chest Computed Tomography $>50 \%$

C-reactive protein $>8$

D-Dimers > 1250

Nasal oxygen catheter

Hudson mask

Azithromycin+Corticosteroid+Anticoagulant

Hydroxych loroquine

Medication interaction

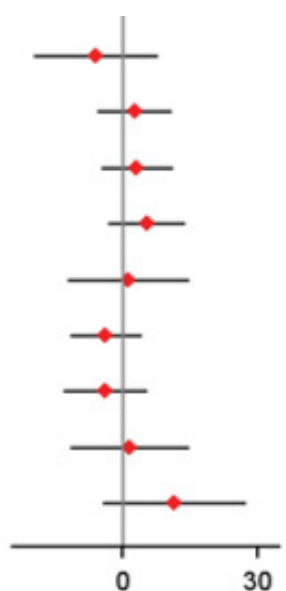

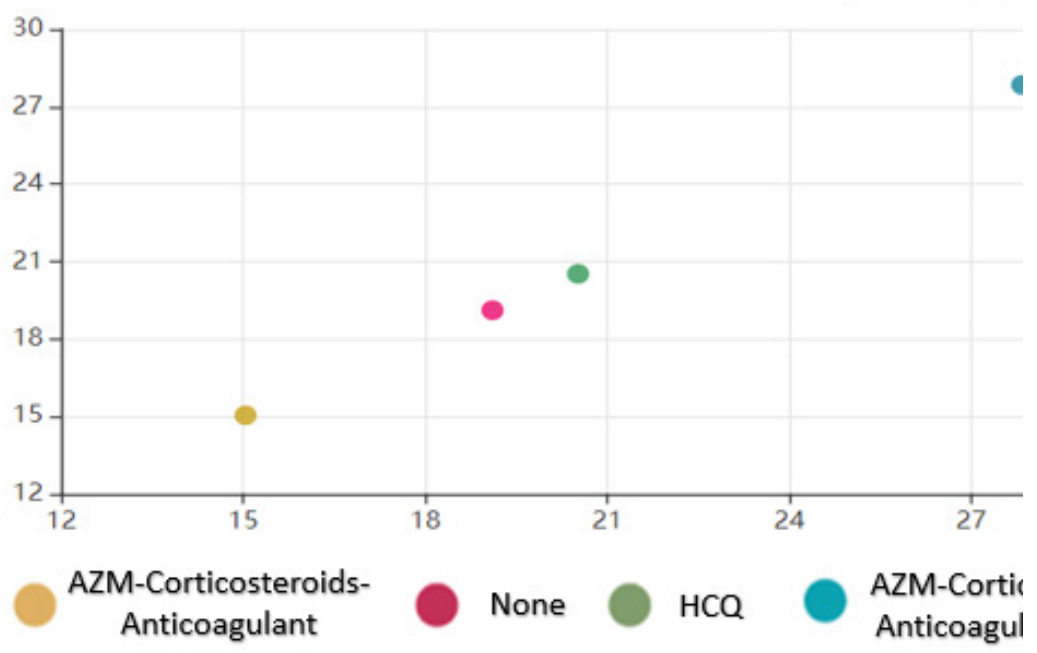

Figure 3: Medication interaction is the behavior of the combination AZM-Corticosteroids-Anticoagulant in the presence or absence of $\mathrm{HCQ}$. At the bottom, the mean length of stay for each outcome according to the combination of treatment use. 
AZM-Corticosteroid and therapeutic anticoagulation when indicated, reduced the mean length of stay in ICU and MV; 3) The introduction of HCQ to the AZMCorticosteroid combination increased the mean length hospital stay; 4) The use of HFNC prevented in one third the patient's progression to MV and 5) Clinical predictors related to higher mortality at admission, included: Age $>65$ years, presence of up one comorbidity, pulmonary involvement > 50\%, saturation < 93\%, lymphopenia, D-dimers and CRP altered.

Observational and randomized clinical trials (RCT) have been published, evaluating the effects of several drugs in terms of potency, efficiency or efficacy in clinical management $[4,5]$. Predictive factors and clinical characteristics that may influence COVID-19 severity have already been demonstrated in the literature and multivariable models have been used to identify highrisk individuals $[6,7]$.

Although some treatments are promising, it is thought to be early to clearly state that there is a definitive treatment. The Solidarity Trial Consortium [8], funded by the World Health Organization showed that antiviral drugs including remdesivir, $\mathrm{HCQ}$, lopinavir, and interferon regimens had little or no effect on hospitalized patients with COVID-19, as indicated by overall mortality, initiation of ventilation, and duration of hospital stay. Also, another promising intervention with convalescent plasma, validated in a RCT showed no significant differences in clinical status or overall mortality between patients treated or received placebo [9].

Although controversial, the use of corticosteroid seems to have clinical potential on mortality reduction and need for intubation, provided it is adequate for the treatment regimen and individual clinical characteristics [10-12]. The Randomized Evaluation of COVID-19 Therapy (RECOVERY trial) showed that survival was significantly higher among patients treated with dexamethasone, especially for those requiring invasive intubation [13]. Our data reinforce these findings and highlight the corticosteroid therapeutic effectiveness, especially in reducing the risk of mortality, ICU admission and need for MV when combined with AZM.

Therapeutic or prophylactic anticoagulation when indicated has proven to be an important strategy to the COVID-19 treatment framework, reducing intubation and mortality [14]. We also observed some benefits related to the reduction of ICU and MV length of stay in patients who used anticoagulant.

Some studies published at the beginning of the pandemic, with limited evidence, highlighted the benefits of using $\mathrm{HCQ}$ combined or not with AZM in reducing mortality and total length of stay [15-16]. However, in an open-label, multicenter, randomized, controlled trial conducted by the Coalition COVID-19 Brazil I, among in patients with mild-to-moderate COVID-19, the use of $\mathrm{HCQ}$, alone or with AZM, did not improve clinical status at 15 days as compared with standard care [17]. Self, et al. reported similar ineffectiveness in $\mathrm{HCQ}$ treatment on the $14^{\text {th }}$ day of hospitalization [18].

Rosenberg and Magagnoli also performed a protocol using HCQ combined or not with AZM and found no reduction in mortality risk and need for MV. Also, they reported an increase in overall mortality for patients treated with $\mathrm{HCQ}$ alone. In the same direction, we observed that whenever $\mathrm{HCQ}$ was included in the model, the protective benefit of the association of AZMCorticosteroids loses significance and becomes a risk factor for a worse prognosis $[19,20]$. We showed that patients treated with $\mathrm{HCQ}$ have a longer hospitalization compared to patients not treated; a finding previously discussed by Kalligeros, et al. [21]. Interestingly also, when we analyzed the clinical predictors influence under the use of $\mathrm{HCQ}$, there was no significant difference between those treated or not with $\mathrm{HCQ}$. We assume that other factors, such drug interactions may be involved in these findings. Besides that, oseltamivir, convalescent plasma, vasopressor and tocilizumab when evaluated alone or combined with $\mathrm{HCQ}, \mathrm{AZM}$ and corticosteroids showed no benefit.

The use of HFNC showed a trend toward reduction in the intubation rate and no difference in mortality, findings similar to those reviewed by Lin [22]. Geng, presented HFNC as a favorable option to avoid intubation through adequate monitoring of the respiratory function of COVID patients [23].

Clinical predictors associated with mortality included individuals older than > 65 years, with up to one comorbidity, pulmonary involvement more than $50 \%$, saturation < 93\%, Iymphopenia, elevated D-dimers and CRP at admission. Oxygen requirement through BiPAP or HFNC, ICU admission and MV required during hospitalization were also risk markers. A recent publication showed that patients with leukocytosis and CRP altered on arrival were associated with poor prognosis and may predict the severity of COVID-19 [24]. Other reports also found the increase of biochemical and inflammatory markers-D-dimers, ferritin, lactate dehydrogenase, and CRP-when no anticoagulant was used in treatment [14].

The weakness of our study is related to the fact that it is observational, unicentric and retrospective however our results are in line with other RCTs that recommended the association of corticosteroids to the set of treatment and advise against $\mathrm{HCQ}$ use in patients with COVID-19. The reduced HCQ treatment efficiency when included to the set of drugs can be speculate through the pharmacological interaction with others drugs triggering for example an increase in its serum concentration, prolongation of the QT interval in the ECG and possibly triggering episodes of 
ventricular tachycardia [25-28]. It is known that longer QTc can cause life-threatening arrhythmias especially in critically ill patients, however monitoring of ECG and drug serum level was not uniformly standardized, given the retrospective nature of the study. Finally, the results of our study should be evaluated considering individual clinical characteristics in a real world and clinicians should carefully weigh the risks and benefits when considering any therapeutic scheme out of the randomized clinical trial setting.

\section{Conclusion}

In this retrospective cohort study, AZMCorticosteroids and therapeutic anticoagulation, when indicated, represented a favorable combination for patients hospitalized with COVID-19, reducing mortality, length of hospitalization and the risk of MV. $\mathrm{HCQ}$ did not yield benefits to combination therapy and we do not support its use for in patients. HFNC oxygen therapy was able to reduce the risk of MV support. Individuals older than $>65$ years, with presence of up one comorbidity, pulmonary involvement more than $50 \%$, saturation $<93 \%$, lymphopenia, D-dimers and CRP elevated on arrival, oxygen requirement through BiPAP or HFNC, ICU admission and MV during hospitalization represented the set of clinical predictors for worse prognosis.

\section{Declarations of Interest}

None.

\section{References}

1. Robert V, Lucy CO, Ilaria D, Peter W, Charles W, et al (2020) Estimates of the severity of coronavirus disease 2019: A model-based analysis. Lancet Infect Dis 20: 669677.

2. Infectious Diseases Society of America Guidelines on the treatment and management of patients with covid-19.

3. Carisi AP, Vania R, Gisele NB, Alexandre PZ, Luis AN, et al. (2020) Getting ready for the covid-19 pandemic: Experience of a Brazilian Hospital. N Engl J Med.

4. Maicon F, Verônica C, Cinara S, Luciano CPA, Angela $\mathrm{MB}$, et al. (2020) Guidelines for the pharmacological treatment of covid-19. The task-force/consensus guideline of the Brazilian Association of Intensive Care Medicine, the Brazilian Society of Infectious Diseases and the Brazilian Society of Pulmonology and Tisiology. Rev Bras Ter Intensiva 32: 166-196.

5. Wise J, Coombes R (2020) Covid-19: The inside story of the recovery trial. BMJ 370: $\mathrm{m} 2670$.

6. Barda N, Riesel D, Akriv A, Levy J, Finkel U, et al. (2020) Developing a covid-19 mortality risk prediction model when individual-level data are not available. Nat Commun 11: 4439.

7. Zhao Z, Chen A, Hou W, James MG, Haifang Li, et al. (2020) Prediction model and risk scores of ICU admission and mortality in covid-19. PLoS One 15: e0236618.

8. WHO Solidarity Trial Consortium (2020) Repurposed antiviral drugs for covid-19-interim.WHO solidarity trial results. N Engl J Med 384: 497-511.

9. Simonovich VA, Burgos Pratx LD, Scibona P, María VB, Marcelo GV, et al. (2020) A randomized trial of convalescent plasma in covid-19 severe pneumonia. $\mathrm{N}$ Engl J Med 384: 619-629.

10. Fang X, Mei Q, Yang T, Li L, Wang Y, et al. (2020) Lowdose corticosteroid therapy does not delay viral clearance in patients with covid-19. J Infect 81: 147-178.

11. Shang L, Zhao J, Hu Y, Du R, Cao B (2020) On the use of corticosteroids for 2019-nCoV pneumonia. Lancet 395: 683-684.

12. World Health Organization. Corticosteroids for covid-19 guideline.

13. De Backer D, Azoulay E, Vincent JL (2020) Corticosteroids in severe covid-19: A critical view of the evidence. Crit Care 24: 627 .

14. Nadkarni GN, Lala A, Bagiella E, Helena LC, Pedro RM, et al. (2020) Anticoagulation, bleeding, mortality, and pathology in Hospitalized patients with covid-19. J Am Coll Cardiol 76: 1815-1826.

15. Elif T, Mustafa Ç, Binnaz ZY, Efsun GUC, Demet T, et al. (2020) Hydroxychloroquine plus azithromycin and early hospital admission are beneficial in Covid-19 patients: Turkish experience with real-life data. Turk J Med Sci 51: 10-15.

16. Arshad S, Kilgore P, Chaudhry ZS, Gordon J, Dee DW, et al. (2020) Treatment with hydroxychloroquine, azithromycin, and combination in patients hospitalized with covid-19. Int $J$ Infect Dis 97: 396-403.

17. Alexandre BC, Fernando GZ, Regis GR, Luciano CPA, Viviane CV, et al. (2020) Hydroxychloroquine with or without azithromycin in mild-to-moderate covid-19. N Engl J Med 383: 2041-2052.

18. Wesley HS, Matthew WS, Lindsay ML, Jonathan DC, Derek CA, et al. (2020) Effect of hydroxychloroquine on clinical status at 14 days in hospitalized patients with covid-19: A randomized clinical trial. JAMA 324: 2165-2176.

19. Eli SR, Elizabeth MD, Tomoko U, Larissa AW, Jessica K, et al. (2020) Association of treatment with hydroxychloroquine or azithromycin with in-Hospital mortality in patients with covid-19 in New York State. JAMA 323: 2493-2502.

20. Joseph M, Siddharth N, Felipe P, Tammy HC, James WH, et al. (2020) Outcomes of hydroxychloroquine usage in United States Veterans Hospitalized with Covid-19. Med (NY) 1: 114-127.

21. Markos K, Fadi S, Eleftheria A, Evangelia KM, Su A, et al. (2020) Hydroxychloroquine use in hospitalised patients with covid-19: An observational matched cohort study. J Glob Antimicrob Resist 22: 842-844.

22. Lin SM, Liu KX, Lin ZH, Lin PH (2017) Does high-flow nasal cannula oxygen improve outcome in acute hypoxemic respiratory failure? A systematic review and meta-analysis. Respir Med 131: 58-64.

23. Geng S, Mei Q, Zhu C, Yang T, Yang Y, et al. (2020) High flow nasal cannula is a good treatment option for covid-19. Heart Lung 49: 444-445.

24. Takayuki Y, Mako W, Takahiro Y, Nitin C, Takahisa M, et al. (2020) Value of leukocytosis and elevated C-reactive protein in predicting severe coronavirus 2019 (covid-19): A systematic review and meta-analysis. Clin Chim Acta 509: 235-243. 
25. Huang HD, Jneid H, Aziz M, Venkatesh R, Parikshit SS, et al. (2020) Safety and effectiveness of hydroxychloroquine and azithromycin combination therapy for treatment of hospitalized patients with Covid-19: A propensity-matched study. Cardiol Ther 9: 523-534.

26. Nicholas JM, Christina FY, David JS, Timothy RM, Christopher MM, etal. (2020) Risk of QT interval prolongation associated with use of hydroxychloroquine with or without concomitant azithromycin among hospitalized patients testing positive for coronavirus disease 2019 (covid-19). JAMA Cardiol 5: 1036-1041.
27. Chorin E, Wadhwani L, Magnani S, Dai M, Shulman E, et al. (2020) QT interval prolongation and torsade de pointes in patients with covid-19 treated with hydroxychloroquine/ azithromycin. Heart Rhythm 17: 1425-1433.

28. Saleh M, Gabriels J, Chang D, Kim BS, Mansoor A, et al. (2020) Effect of chloroquine, hydroxychloroquine, and azithromycin on the corrected qt interval in patients with sars-cov-2 infection. Circ Arrhythm Electrophysiol 13: e008662. 